\title{
Protective Effect and Mechanisms of Radix Astragali Injection on the Intestinal Mucosa of Rats with Obstructive Jaundice
}

\author{
Zhang Xiping, ${ }^{1}$ Weng Ke, ${ }^{2}$ Yu Yaping, ${ }^{2}$ Zhao Hongchan, ${ }^{3}$ and Cheng Qihui ${ }^{4}$ \\ ${ }^{1}$ Department of General Surgery, Hangzhou First People's Hospital, Zhejiang Province, Hangzhou 310006, China \\ ${ }^{2}$ College of Clinical Medicine, Zhejiang Traditional Chinese Medicine University, Zhejiang Province, Hangzhou 310053, China \\ ${ }^{3}$ Department of Testing, Hangzhou First People's Hospital, Zhejiang Province, Hangzhou 310006, China \\ ${ }^{4}$ Department of Gynaecology and Obstetrics, Hangzhou First People's Hospital, Zhejiang Province, Hangzhou 310006, China
}

Correspondence should be addressed to Zhang Xiping, zxp99688@vip.163.com

Received 9 May 2009; Accepted 6 January 2010

Academic Editor: Francesco Marotta

Copyright ( 2010 Zhang Xiping et al. This is an open access article distributed under the Creative Commons Attribution License, which permits unrestricted use, distribution, and reproduction in any medium, provided the original work is properly cited.

\begin{abstract}
Objective. To research the protective effects and mechanisms of Radix Astragali injection on the intestinal mucosa of rats with obstructive jaundice (OJ). Methods. The rats were randomly divided into sham-operated, model control and Radix Astragali treated group. We observed the pathological changes of intestinal mucosa, expression levels of Bax and NF- $\kappa \mathrm{B}$ proteins, and apoptosis indexes in intestinal mucosa as well as serum NO, MDA and SOD contents, respectively, on $7 \mathrm{~d}, 14 \mathrm{~d}, 21 \mathrm{~d}$ and $28 \mathrm{~d}$ after operation. Results. The pathological severity score (on $7 \mathrm{~d}$ and 14d), apoptotic indexes (on 14d) of the intestinal mucosa and serum MDA content (on 14d) of treated group were significantly lower than those in the model control group $(P<.05)$. The serum SOD contents (on all time points) of treated group were significantly higher than those in the model control group $(P<.05)$. The sham-operated group (on 21d) of the product of staining intensity and positive rate of Bax protein was significantly lower than model control group $(P<.05)$. Conclusion. Radix Astragali injection could protect the intestinal mucosa of OJ rats by increasing the content of SOD, reducing the content of MDA, inhibiting the apoptosis and relieving the pathological changes of intestinal mucosa.
\end{abstract}

\section{Introduction}

During obstructive jaundice, the impairment of intestinal mucosal barrier and the translocation of endotoxins and intestinal bacteria can cause intestinal infections and secondary multiple organ injury, which are pivotal for the deterioration of the disease [1-6]. Therefore, it is of great significance to protect the small intestinal mucosa [7]. OJ is sensitive to auxiliary treatment with traditional Chinese medicine. Astragalus injection, as an extract from Radix Astragali, is characterized by low cost, extensive pharmacological effects and few side effects and therefore has unique advantages and prospects in the treatment of OJ. Astragalus injection is one of the famous injections of traditional Chinese medicine. Astragalus injection contains polysaccharide, saponin, flavones, and trace elements [8]. Although some studies $[8,9]$ showed that Astragalus could significantly improve the oxygen free radical-scavenging abilities, inhibit excessive inflammatory response, and thereby mitigate hepatic and renal injury in OJ rats, no study on the protective effects of astragalus on intestinal mucosa was found in domestic and foreign literature. In this study, we investigated the protective effects and mechanisms of Astragalus injection on small intestinal mucosa of OJ rats to provide a theoretical basis for clinical application of this injection.

\section{Materials and Methods}

2.1. Materials. The healthy male SD rats of clean grade, weighing between 270 and $330 \mathrm{~g}$, were provided by the Laboratory Animal Research Center of the Zhejiang University of Traditional Chinese Medicine (Hangzhou, China). The sodium pentobarbital was purchased from Sigma Corporation (Sigma-Aldrich, St. Louis, MO, USA). Radix Astragali injection (each $10 \mathrm{~mL}$ vial contains active components equivalent to $20 \mathrm{~g}$ of the original medicine) was 
purchased from Chiatai Qingchunbao Pharmaceutical Co., Ltd (Hangzhou, China). The serum nitrogen monoxidum (NO), malonaldehyde (MDA), and superoxide dismutase (SOD) kits were all purchased from Nanjing Jiancheng Bioengineering Research Institute (Nanjing, China), and the calculation units for content are respectively $\mu \mathrm{mol} / \mathrm{L}$, $\mathrm{nmol} / \mathrm{mL}$, and $\mathrm{U} / \mathrm{mL}$. The anti-NF- $\kappa \mathrm{B}$ P65 and anti-Bax antibody were purchased from Santa Cruz Biotechnology, Inc. (Santa Cruz, California, USA) TUNEL assay kit was purchased from Takara Bio Inc. (Jingdu, Japan).

\subsection{Methods}

2.2.1. Animal Grouping and Preparation of OJ Models. 180 rats were utilized for OJ-associated experiments and randomly divided into sham-operated, model control, and treated group ( $n=60)$, which were further randomly subdivided into $7 \mathrm{~d}, 14 \mathrm{~d}, 21 \mathrm{~d}$, and $28 \mathrm{~d}$ groups $(n=$ 15) according to time duration after operation. After rats were anesthetized with an intraperitoneal injection of $2.5 \%$ sodium pentobarbital $(0.2 \mathrm{~mL} / 100 \mathrm{~g})$, the abdominal cavity was opened to identify and dissociate common bile duct along the hepatoduodenal ligament. For rats in the model control group and the treated group, the proximal end of common bile duct was double ligated with surgical threads, common bile duct was cut off, and a layered suture of the abdominal wall was performed to close the abdominal cavity. For rats in the sham-operated groups, common bile duct was only dissociated but not ligated, and a layered suture of the abdominal wall was also performed to close the abdominal cavity. An intraperitoneal injection of Radix Astragali injection at a dose of $0.75 \mathrm{~mL} / 100 \mathrm{~g} / \mathrm{d}$ was given to rats in the treated groups while equal volume of physiological saline solution was used in the sham-operated and the model control group. Different injection was maintained until the end of the 7-day, 14-day, 21-day, and 28-day observation period in the corresponding groups [8].

2.2.2. Determination of Experimental Parameters. The mortality rate of rats in each group was recorded. After mercy killing rats anesthetized by sodium pentobarbital in batches, we collected the serum of rats to detect NO, MDA and SOD contents and observed the pathological changes of intestinal muscoa. The determination of these serum parameters was conducted according to the instructions provided by the kits. The pathological severity score of intestinal muscoa was conducted according to our report for related standard. And then we prepared the tissue microarrys section for intestine tissue with diameter of $1.5 \mathrm{~mm}$, stained them, observed the changes in the expression levels of Bax and NF- $\kappa \mathrm{B}$ P65 proteins as well as the apoptosis index of intestinal muscoa.

\subsubsection{Immunohistochemical Staining of Bax and NF- $\kappa B P 65$} Proteins in the Intestinal Muscoa. Envision two-step method was used to detect the expression levels of Bax and NF- $\kappa$ B P65 protein in the intestinal muscoa. The evaluation standard was as follows: (1) the staining intensity was evaluated according to the extent of cell coloration: "-" represented negative staining; “+” represented mild staining, positively stained cells showed a yellow pigment; " ++ " represented moderate staining, positively stained cells showed a brown pigment; “+++" represented intense staining, positively stained cells showed a dark brown pigment, each of which was scored as $0,1,2$, and 3 points, respectively, during statistical analysis; (2). the evaluation standard of the positive rate: there was no positive cells $(-)$; the percentage of positive cells was less than $25 \%(+)$; the percentage of positive cells ranged between $26 \%$ and $50 \%(++)$;the percentage of positive cells was more than $50 \%(+++)$, each of which was scored as $0,1,2$, and 3 points, respectively, during statistical analysis.

2.2.4. TUNEL Staining in the Intestinal Muscoa. In tissue microarrays sections, DNA nick in situ end-labeling (TUNEL) staining steps were performed as follows: baking sections under $60^{\circ} \mathrm{C}$ for 30 minutes, routine deparaffinage, and Milli-Q wash for 5 minutes. Processing tissue with Protease K $(10 \mathrm{ug} / \mathrm{uL})$ under room temperature for 15 minutes, PBS washes for 5 minutes. Using $3 \% \mathrm{H}_{2} \mathrm{O}_{2}$ solution to block endogenous peroxydase for 5 minutes, PBS wash for 5 minutes $\times$ twice. Adding $30 \mathrm{uL}$ reaction solution in freezing condition (TdT Enzyme: Labeling Safe Buffer $=1: 10$ ), $37^{\circ} \mathrm{C}$ incubation for 90 minutes, PBS wash for 5 minutes $\times$ twice. Adding $50 \mathrm{ul}$ Anti-FITC HRP Conjugate, $37^{\circ} \mathrm{C}$ incubation for 30 minutes, $\mathrm{PBS}$ wash for 5 minutes $\times$ twice. $\mathrm{DAB}$ coloration, Milli-Q wash to terminate coloration. Hematoxylin counterstain, water wash and wash fully with water after differentiation till return blue; routine dehydration and transparence; neutral gum mounting. The apoptotic index was calculated. Apoptotic index $=$ apoptotic cell count/total cell count $\times 100 \%$.

2.2.5. Statistical Analysis. After input into the Excel sheets, the compiled data was read into SPSS15.0 for further analysis. Normal data was expressed as means (standard deviation) while nonnormal data were expressed as medians (interquartile range). Analysis of variance and pairwise comparisons were used in normal data; whereas nonnormal data were subjected to nonparametric test, among which Kruskal-Wallis $H$ test was used for pairwise comparisons and Mann-Whitney $U$ test for multiple comparisons. Yates' chi-square test $\left(\chi^{2}\right)$ was used for intergroup comparisons of mortality rates.

\section{Results}

3.1. Comparison of Mortality Rate. All rats were alive in the sham-operated groups on all time points after operation. 2 and 1 rats died in the model control and treated group on $7 \mathrm{~d}$, respectively; 4 and 3 rats died in the model control and treated group on 14d, respectively; 4 rats died in both model control and treated group on 21d; 7 and 6 rats died in the model control and treated group on $28 \mathrm{~d}$, respectively. The total mortality rate of the model control and treated group 


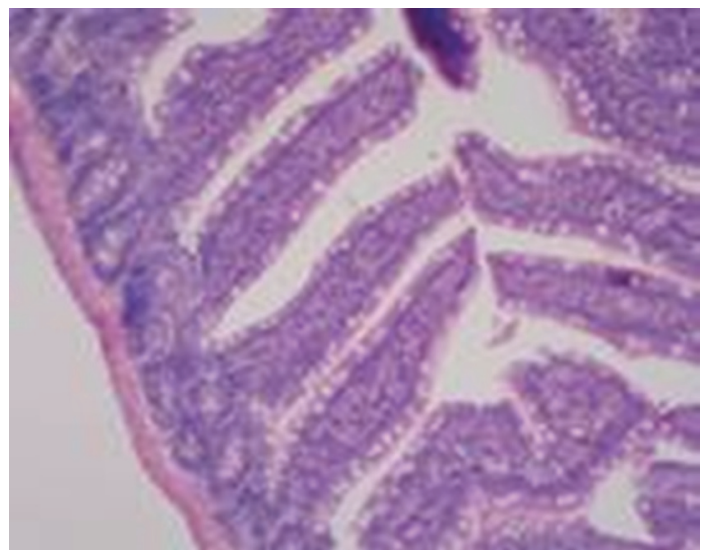

Figure 1: Sham-operated group-28d mucous membrane of small intestine is approximately normal $\mathrm{HE} \times 100$.

on $28 \mathrm{~d}$ were significantly higher than those in the shamoperated group $(P<.001)$. There was no marked difference between the model control and treated group $(P>.05)$.

\subsection{Pathological Changes in Intestinal Mucosa}

\subsubsection{Sham-Operated Group}

Gross Pathological Changes. No obvious abnormality was seen.

Pathological Changes under Light Microscopy. No obvious difference in pathological changes was observed among each time point after operation. The intestinal mucosa was normal in the majority of rats. The intestinal mucosal epithelium was not intact and inflammatory cell infiltration of proper layer was seen in very few rats (see Figure 1).

\subsubsection{Model Control Group}

Gross Pathological Changes. On 7d after operation, intestinal wall and peritoneum became jaundice in the majority of rats. On 14d after operation, varying degrees of yellow staining of the intestinal wall and peritoneum were seen in the majority of rats. The intestinal canal was enlarged and showed fluid retention. On 21 and 28d after operation, yellow staining of the intestinal wall and peritoneum were seen in all rats.

Pathological Changes under Light Microscopy. No obvious difference in pathological changes was observed among each time point after operation. On $7 \mathrm{~d}$ after operation, intestinal mucosa was normal in the majority of rats, and the edema of submucous layer were present in very few rats. On 14d after operation, intestinal mucosa was normal in the majority of rats but not intact in some rats, and the edema of proper layer, submucous layer, and serosal layer were seen in some rats. On 21d after operation, intestinal mucosa was not intact in the majority of rats, the edema of proper layer, submucous layer, and serosal layer were seen in the majority of rats, and very few rats showed no abnormality of the intestinal

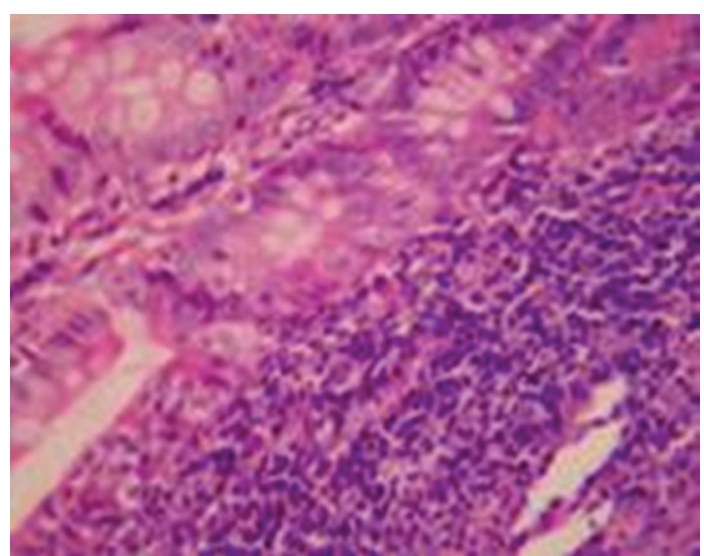

Figure 2: Model control group-28d a large inflammatory cells infiltration in small intestinal wall $\mathrm{HE} \times 200$.

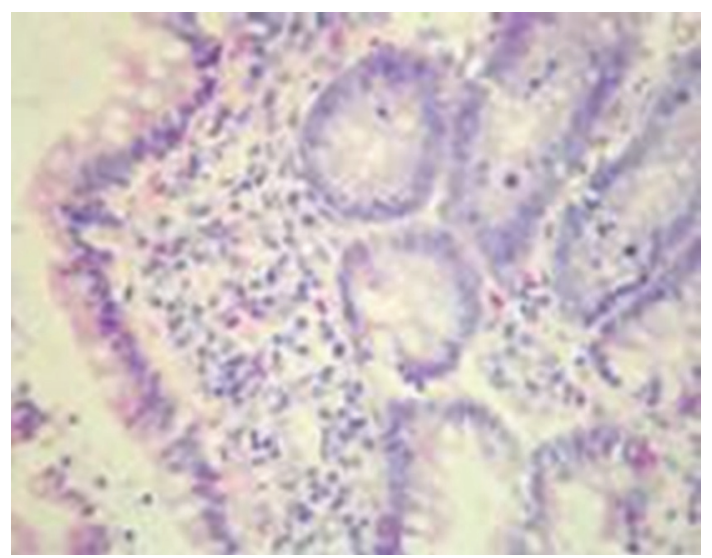

FIGURE 3: Model control group-28d a small quantity acidophile cells infiltration in intestinal lamina propria $\mathrm{HE} \times 200$.

mucosa. On 28d after operation, focal necrosis in intestinal mucosal epithelium as well as the edema of proper layer, submucous layer and serosal layer were seen in the majority of rats (see Figures 2 and 3 ).

\subsubsection{Treated Group}

Gross Pathological Changes. On 7d after operation, no obvious difference was observed when compared to that in model control group. On $14 \mathrm{~d}$ after operation, intestinal wall became jaundice in half of the rats, but intestinal canal was not enlarged and showed no fluid retention. On 21, and 28d after operation, no obvious difference was observed when compared to those in model control group.

Pathological Changes under Light Microscopy. The pathological changes in the small intestine of rats at various time points showed varying degrees of mitigation. On day 7 in treated group, the small intestine of the majority of rats showed no abnormality while the small intestine of extremely few rats showed inflammatory cell infiltration in proper layer, submucous layer, and serosal layer. On day 14 
TABLE 1: Comparison of different pathological indexes $\left(M\left(Q_{R}\right)\right)$.

\begin{tabular}{|c|c|c|c|c|}
\hline Index & Time & Sham-operated group & Model control group & Treated group \\
\hline \multirow{4}{*}{ Pathological severity score } & $7 d$ & $0.0(1.0)$ & $1.0(1.0)^{*}$ & $0.0(1.0)^{*+}$ \\
\hline & $14 \mathrm{~d}$ & $0.0(1.0)$ & $1.0(2.0)^{*}$ & $1.0(1.0)^{+}$ \\
\hline & $21 d$ & $0.0(0.0)$ & $2.0(1.0)^{*}$ & $0.0(1.0)$ \\
\hline & $28 \mathrm{~d}$ & $0.0(0.0)$ & $2.0(1.0)^{*}$ & $1.0(0.0)$ \\
\hline \multirow{4}{*}{ Product of the staining intensity and positive rate of Bax } & $7 \mathrm{~d}$ & $0.0(0.0)$ & $0.0(1.0)$ & $0.0(1.0)$ \\
\hline & $14 \mathrm{~d}$ & $0.0(1.0)$ & $2.0(4.0)$ & $0.0(1.5)$ \\
\hline & $21 \mathrm{~d}$ & $0.0(0.0)^{+}$ & $1.0(2.0)$ & $0.0(1.0)$ \\
\hline & $28 \mathrm{~d}$ & $0.0(1.0)$ & $0.5(1.5)$ & $1.0(2.0)$ \\
\hline \multirow{4}{*}{ Apoptosis index } & $7 \mathrm{~d}$ & $0.0(0.0)^{+}$ & $0.0(0.0)$ & $0.0(0.0)$ \\
\hline & $14 \mathrm{~d}$ & $0.0(0.0)$ & $0.0(0.01)$ & $0.0(0.0)^{+}$ \\
\hline & $21 d$ & $0.0(0.0)^{+}$ & $0.0(0.01)$ & $0.0(0.0)$ \\
\hline & $28 \mathrm{~d}$ & $0.0(0.0)^{+}$ & $0.0(0.0)$ & $0.0(0.0)$ \\
\hline \multirow{4}{*}{ Product of the staining intensity and positive rate of NF- $\kappa$ B } & $7 \mathrm{~d}$ & $0.0(0.0)$ & $0.0(2.0)$ & $0.0(2.0)$ \\
\hline & $14 \mathrm{~d}$ & $0.0(2.0)$ & $0.0(4.0)$ & $0.0(1.0)$ \\
\hline & $21 \mathrm{~d}$ & $0.0(0.0)$ & $0.0(4.0)$ & $0.0(3.0)$ \\
\hline & $28 \mathrm{~d}$ & $0.0(2.0)$ & $1.0(3.0)$ & $0.0(1.0)$ \\
\hline
\end{tabular}

Note: Compare to sham-operated group, ${ }^{*} P<.05$ and ${ }^{* *} P<.01$; Compare to model control group, ${ }^{+} P<.05$ and ${ }^{++} P<.01$.

in treated group, the small intestine of some rats showed no abnormality, the small intestine of some rats showed inflammatory cell infiltration in proper layer, submucous layer, and serosal layer, and the small intestine of extremely few rats showed focal necrosis in the mucosa. On day 21 in treated group, the small intestine of some rats showed no abnormality while the other rats showed inflammatory cell infiltration in proper layer, submucous layer and serosal layer. On day 28 in treated group, the small intestine of some rats showed inflammatory cell infiltration in proper layer, submucous layer and serosal layer while the extremely few rats showed no abnormality (see Figure 4).

3.3. Comparison of the Pathological Severity Scores. The pathological score standard of intestinal mucosa was referred the report [10]. Pathological severity scores of the shamoperated group (on 7, 14, and 21d) was significantly lower than model control group $(P<.05)$. The sham-operated group (on $7 \mathrm{~d}$ ) were significantly lower than treated group $(P<.05)$. The scores in treated group (on 7 and $14 \mathrm{~d})$ were significantly lower than those in model control group $(P<.05)$ see Table 1.

3.4. Comparison of the Product of Staining Intensity and Positive Rate of NF- $\kappa B$ Protein. The positive signals for $\mathrm{NF}-\kappa \mathrm{B}$ protein were mainly localized in the cytoplasm of intestinal mucosa epithelial cells and occasionally in the cytoplasm of inflammatory cells. The positive signals were mainly localized in the cytoplasm though few were seen in the nucleus. On all time points after operation, no marked difference was noted among all groups $(P>.05)$; see Table 1 , Figures 5 and 6.

3.5. Comparison of the Product of Staining Intensity and Positive Rate of Bax Protein. The sham-operated group (on 21d) was significantly lower than model control group $(P<$ $.05)$; see Table 1, Figures 7, 8, 9, and 10.

3.6. Comparison of the Apoptosis Index. The sham-operated group (on 7, 21 and 28d) was significantly lower than model control group $(P<.05)$. The treated group (on 14d) was significantly lower than model control group $(P<.05)$; see Table 1, Figure 11.

3.7. Comparison of Serum MDA. The sham-operated group (on all time points) was significantly lower than model control and treated group $(P<.01)$. The treated group (on 14d) was significantly lower than model control group $(P<.05)$; see Table 2 .

3.8. Comparison of Serum SOD. The sham-operated group (on all time points) was significantly higher than model control and treated group $(P<.05)$. The treated group (on all time points) was significantly higher than model control group $(P<.05)$; see Table 2 .

3.9. Comparison of Serum NO. The sham-operated group (on 7 and 14d) was significantly lower than model control and treated group $(P<.01)$; see Table 2 .

\section{Discussion}

The intestine is the largest lymphoid organ of the body. Since the intestinal canal has a constant contact with viruses, bacteria and foreign matters, the surface mucosa of the intestine plays an important role in preventing the invasion of harmful substances. Therefore, protection of small intestinal mucosa is a key to the treatment of OJ. During OJ, highconcentration bile salts and hyperbilirubinemia may be two 
TABLE 2: Comparison of different indexes in blood $(\bar{X} \pm S)$.

\begin{tabular}{|c|c|c|c|c|}
\hline Index & Time & Sham-operated group & Model control group & Treated group \\
\hline \multirow{4}{*}{ MDA } & $7 d$ & $2.5 \pm 1.2$ & $21.6 \pm 3.2^{* *}$ & $22.8 \pm 4.8^{* *}$ \\
\hline & $14 \mathrm{~d}$ & $2.3 \pm 1.4$ & $23.4 \pm 3.4^{* *}$ & $19.6 \pm 7.2^{* *++}$ \\
\hline & $21 d$ & $3.1 \pm 1.7$ & $27.1 \pm 4.7^{* *}$ & $21.0 \pm 6.2^{* *}$ \\
\hline & $28 \mathrm{~d}$ & $3.6 \pm 1.0$ & $34.0 \pm 9.2^{* *}$ & $27.0 \pm 12.3^{* *}$ \\
\hline \multirow{4}{*}{ SOD } & $7 \mathrm{~d}$ & $56.87 \pm 12.62$ & $31.27 \pm 12.77^{*}$ & $43.61 \pm 8.77^{*+}$ \\
\hline & $14 \mathrm{~d}$ & $53.68 \pm 9.14$ & $32.16 \pm 8.39^{*}$ & $45.28 \pm 11.18^{*+}$ \\
\hline & $21 \mathrm{~d}$ & $57.93 \pm 12.77$ & $34.25 \pm 12.08^{*}$ & $46.38 \pm 16.81^{*+}$ \\
\hline & $28 \mathrm{~d}$ & $57.90 \pm 7.83$ & $34.95 \pm 9.76^{*}$ & $46.88 \pm 11.69^{*+}$ \\
\hline \multirow{4}{*}{$\mathrm{NO}$} & $7 \mathrm{~d}$ & $24.0 \pm 11.0$ & $36.0 \pm 27.0^{* *}$ & $30.0 \pm 17.0^{* *}$ \\
\hline & $14 \mathrm{~d}$ & $25.0 \pm 10.0$ & $47.0 \pm 32.0^{* *}$ & $37.0 \pm 17.0^{* *}$ \\
\hline & $21 d$ & $26.0 \pm 13.0$ & $48.0 \pm 14.0$ & $44.0 \pm 16.0$ \\
\hline & $28 \mathrm{~d}$ & $29.0 \pm 8.0$ & $69.0 \pm 28.0$ & $50.0 \pm 15.0$ \\
\hline
\end{tabular}

Note: Compare to sham-operated group, ${ }^{* *} P<.05$ and ${ }^{*} P<.01$; Compare to model control group, ${ }^{+} P<.05$ and ${ }^{++} P<.01$.

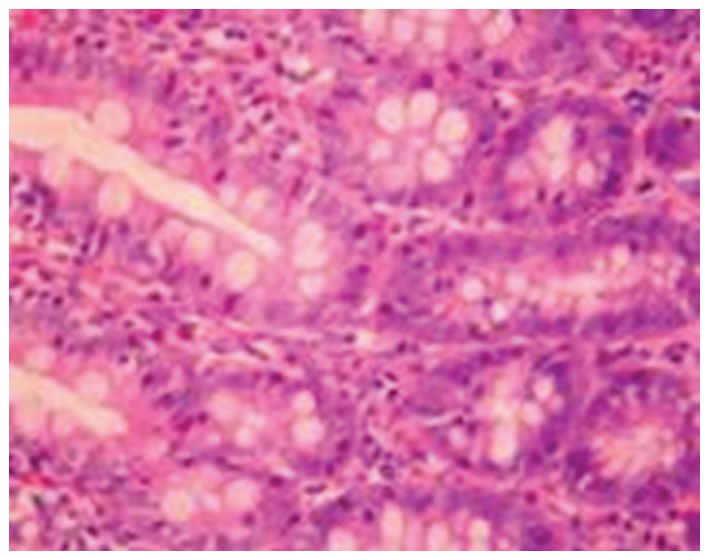

FIGURE 4: Treated group-28d mucous membrane of small intestine is approximately normal $\mathrm{HE} \times 200$.

major factors contributing to the production of oxygen free radicals. Thus, inhibition of hyperbilirubinemia can reduce OJ-induced injury $[11,12]$. To a certain extent, MDA is a reliable parameter that can reflect, to a certain extent, oxygen free radical-induced lipid peroxidation and the extent of lipid peroxidation-induced damage to the body. SOD is able to specifically scavenge superoxide anion radicals and thereby exerts some protective effects on the body. This study indicated that the content of serum MDA in astragalus treated group was significantly lower than that in model control group, the content of SOD in astragalus treated group was significantly higher than that in model control group, and the pathological injury of small intestinal mucosa showed varying degrees of mitigation, suggesting that astragalus can reduce the content of serum MDA, increase the content of SOD and mitigate lipid peroxidation-induced cytoplasmic membrane damage, thereby exert protective effects on the small intestinal mucosa. Moderate NO levels are able to expand blood vessels and improve microcirculation, while high levels of NO can aggravate OJ-induced haemodynamic

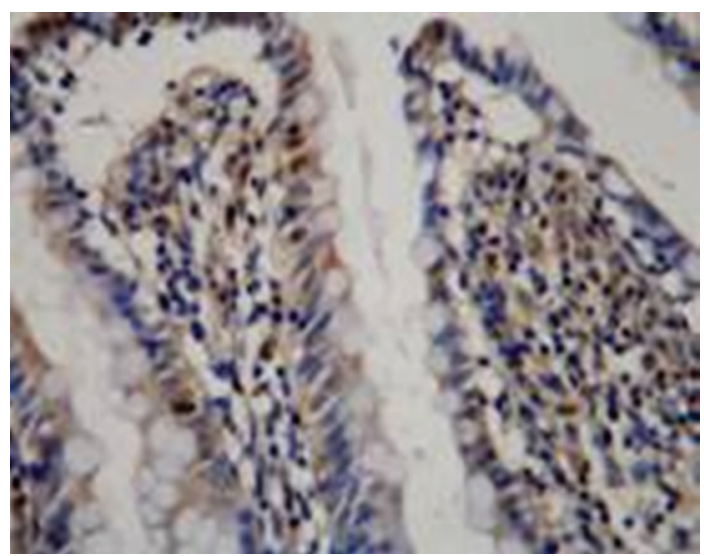

FIGURE 5: Model control group-28d mucous membrane of small intestine $(+++) \mathrm{NF}-\kappa \mathrm{B} \times 200$.

disturbance in the liver and kidney. Han et al. [13] proved that iNOS had an elevated level in the liver of OJ rats and thus could induce the production of a large amount of $\mathrm{NO}$ and cause endothelial cell dysfunction. Our results indicated that, though the level of serum NO in astragalus treated group was lower than that in model control group, no statistical difference was observed. We speculate that it may be due to too small sample size.

Nuclear factor $\kappa \mathrm{B}(\mathrm{NF}-\kappa \mathrm{B})$ is a multieffect transcription regulatory factor that controls the transcription of a variety of inflammation-, immunity-, and hyperplasiarelated factors, such as TNF- $\alpha$, IL- $1 \beta$, iNOS, and ICAM- 1 . Upon activation, $\mathrm{NF}-\kappa \mathrm{B}$ is able to induce a cascade reaction of cytokines [14] and impair the structure and function of multiple organs [15-18]. Some experimental studies showed that NF- $\kappa \mathrm{B}$ may induce or aggravate small intestinal ischemia/reperfusion injury [19], promote intestinal epithelial cell apoptosis [20], and thereby induce intestinal damage. This study showed that at all time points after operation, the expression levels of NF- $\kappa \mathrm{B}$ protein showed no significant 


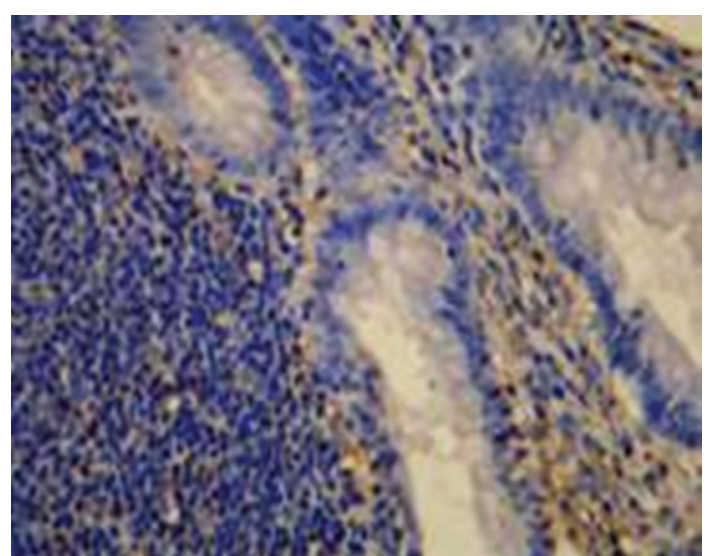

Figure 6: Model control group-28d mucous membrane of small intestine $(++) \mathrm{NF}-\kappa \mathrm{B} \times 200$.

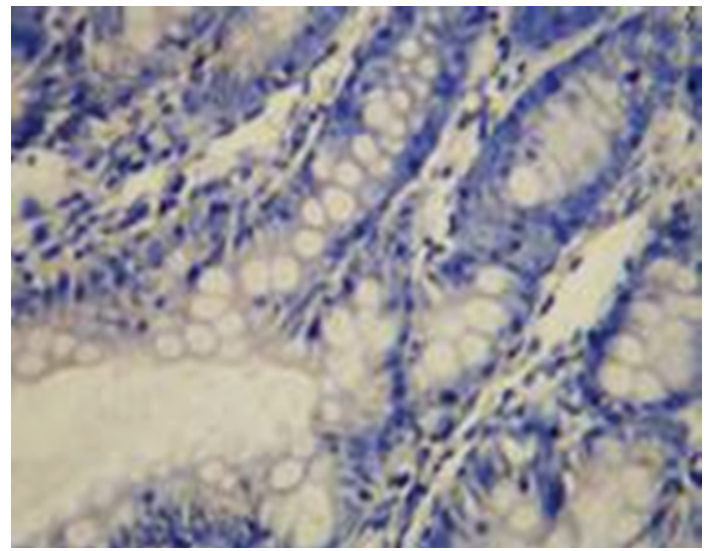

FigURE 7: sham-operated group-21d mucous membrane of small intestine (-) Bax $\times 200$.

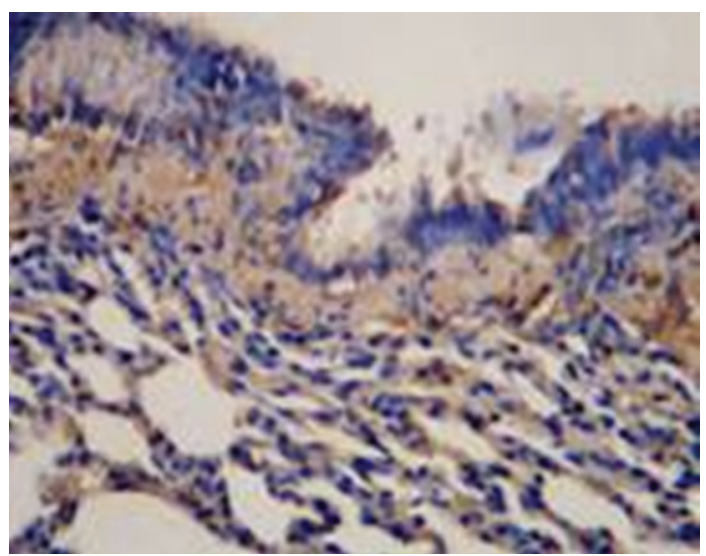

Figure 8: Model control group-21d mucous membrane of small intestine $(+++)$ Bax $\times 200$.

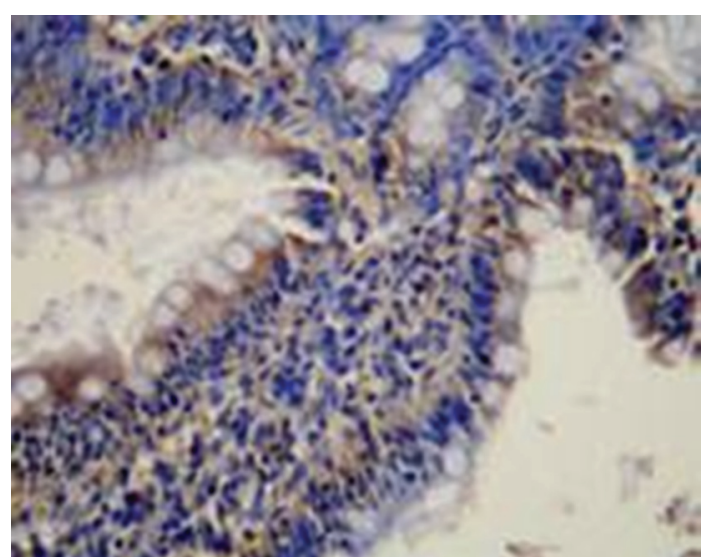

FIGURE 9: Model control group-21d mucous membrane of small intestine $(++)$ Bax $\times 200$.

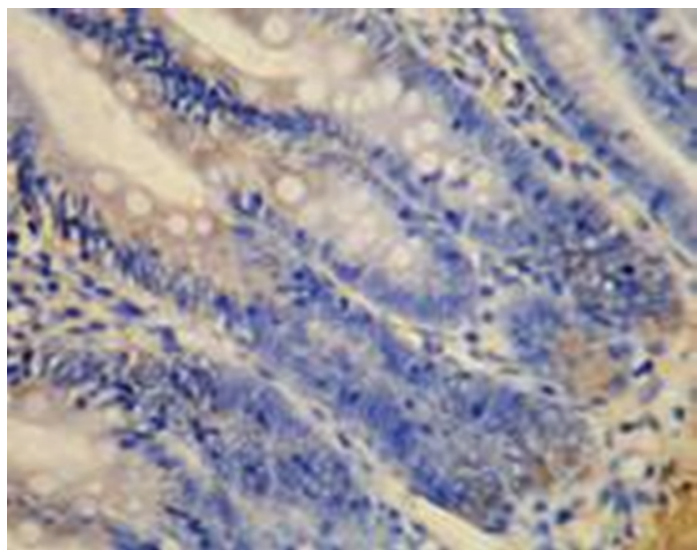

Figure 10: Treated group-21d mucous membrane of small intestine (+) $\mathrm{Bax} \times 200$.

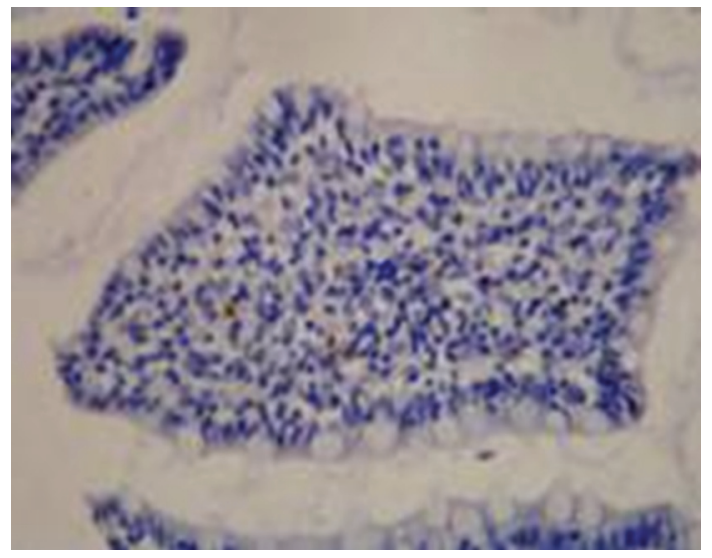

Figure 11: Treated group-21d a few apoptotic cells in mucous membrane of small intestine TUNEL $\times 200$. 
difference among each group $(P>.05)$. So we surmise that $\mathrm{NF}-\kappa \mathrm{B}$ protein has no significant impact on the pathological injury of the small intestine mucosa of OJ rats.

Apoptosis is a kind of gene-controlled physiological cell death to maintain the body's normal physiological functions and stability. The stability of small intestinal mucosa also depends on the equilibrium between the proliferation and apoptosis of epithelial cells [21]. Some factors such as ischemia/reperfusion injury, bacterial infections and nutritional deficiencies may induce the apoptosis of intestinal epithelial cells [22-27] and cause intestinal dysfunction [28, 29]. Yang et al. [30] found that the total extract of astragalus had inhibitory effects on both in vivo and in vitro hepatic cell injury and apoptosis. In the present study, we found that, on day 14, the apoptosis index in small intestinal mucosa in astragalus treated group was significantly lower than that in model control group, and small intestinal mucosal inflammation and necrosis and microvilli defects in treated group were milder than those in model control group, indicating that astragalus can exert protective effects on the small intestinal mucosa of OJ rats through inhibiting the apoptosis of small intestinal mucosal epithelial cells. Bax, as an apoptosis-inducing gene [31-35], can promote Bcl-2 [3638] or Bcl-x dimer formation and thus induce cell apoptosis. In this study, no statistical difference existed in the products of the staining intensity and the positive staining rate of Bax protein at all time points was noted between treated group and model control group, suggesting that Bax protein may have no impact on OJ-induced intestinal mucosa injury and other unknown factors are involved in this process.

In summary, astragalus injection can increase the content of serum SOD, decrease the content of MDA, and inhibit the apoptosis of small intestinal mucosa cells, thereby mitigate the pathological injury of the small intestinal mucosa and exerting protective effects on the small intestinal mucosa of $\mathrm{OJ}$ rats. However, it is worth mentioning that the therapeutic effects of astragalus are limited and auxiliary. Therefore, combined use of other drugs that can protect the small intestinal mucosa is needed during the course of treatment.

\section{Acknowledgments}

This work was supported by technological foundation project of Traditional Chinese Medicine Science of Zhejiang province (no. 2003C130; no. 2004C142), foundation project for medical science and technology of Zhejiang province (no. 2003B134), grave foundation project for technological and development of Hangzhou (no. 2003123B19), intensive foundation project for technology of Hangzhou (no. 2004Z006), foundation project for medical science and technology of Hangzhou (no.2003A004) and foundation project for technology of Hangzhou (no. 2005224).

The authors claimed that this paper was original and would not have any financial interest in a company or its competitor, and that they meet criteria for authorship. They abided the ethics in this animal experiment study. The ethics committee approval of our hospital was secured for the animal study reported, and all rats have not been abused and executive mercy killing when the observing time in this study was over.

\section{References}

[1] V. Bansal and V. D. Schuchert, "Jaundice in the intensive care unit," Surgical Clinics of North America, vol. 86, no. 6, pp. 1495-1502, 2006.

[2] E. A. Beierle, J.-N. Vauthey, L. L. Moldawer, and E. M. Copeland III, "Hepatic tumor necrosis factor- $\alpha$ production and distant organ dysfunction in a murine model of obstructive jaundice," American Journal of Surgery, vol. 171, no. 1, pp. 202-206, 1996.

[3] G. M. Swank and E. A. Deitch, "Role of the gut in multiple organ failure: bacterial translocation and permeability changes," World Journal of Surgery, vol. 20, no. 4, pp. 411-417, 1996.

[4] S. F. Assimakopoulos, C. D. Scopa, and C. E. Vagianos, "Pathophysiology of increased intestinal permeability in obstructive jaundice," World Journal of Gastroenterology, vol. 13, no. 48, pp. 6458-6464, 2007.

[5] W. D. B. Clements, P. Erwin, M. D. McCaigue, I. Halliday, G. R. Barclay, and B. J. Rowlands, "Conclusive evidence of endotoxaemia in biliary obstruction," Gut, vol. 42, no. 2, pp. 293-299, 1998.

[6] Y. F. Tian, Y. Li, Q. Zhao, et al., "Effect of ulinastatin on intestinal mucosal barrier function of rats with obstructive jaundice," Nan Fang Yi Ke Da Xue Xue Bao, vol. 27, no. 7, pp. 987-990, 2007 (Chinese).

[7] E. Gurleyik, O. Coskun, N. Ustundag, and E. Ozturk, "Prostaglandin E1 maintains structural integrity of intestinal mucosa and prevents bacterial translocation during experimental obstructive jaundice," Journal of Investigative Surgery, vol. 19, no. 5, pp. 283-289, 2006.

[8] R.-P. Zhang, X.-P. Zhang, Y.-F. Ruan, et al., "Protective effect of Radix Astragali injection on immune organs of rats with obstructive jaundice and its mechanism," World Journal of Gastroenterology, vol. 15, no. 23, pp. 2862-2869, 2009.

[9] W. M. Feng, Y. Bao, M. Y. Fei, et al., "Perioperative administration of astragalus injection on liver and renal function in patients with obstruction jaundice," Chinese Journal of Surgery of Integrated Traditional and Western Medicine, vol. 10, no. 3, pp. 143-145, 2004 (Chinese).

[10] X. Zhang, L. Chen, L. Luo, et al., "Study of the protective effects of dexamethasone on ileum mucosa injury in rats with severe acute pancreatitis," Pancreas, vol. 37, no. 3, pp. e74-e82, 2008.

[11] R. W. Parks, W. D. B. Clements, M. G. Smye, C. Pope, B. J. Rowlands, and T. Diamond, "Intestinal barrier dysfunction in clinical and experimental obstructive jaundice and its reversal by internal biliary drainage," British Journal of Surgery, vol. 83, no. 10, pp. 1345-1349, 1996.

[12] J. W. Greve, J. G. Maessen, T. Tiebosch, W. A. Buurman, and D. J. Gouma, "Prevention of postoperative complications in jaundiced rats. Internal biliary drainage versus oral lactulose," Annals of Surgery, vol. 212, no. 2, pp. 221-227, 1990.

[13] X. Han, M. P. Fink, T. Uchiyama, R. Yang, and R. L. Delude, "Increased iNOS activityis essential for hepatic epithelial tight junction dysfunction in endotoxemic mice," American Journal of Physiology, vol. 286, no. 1, pp. 126-136, 2004.

[14] M. Ogawa, "Acute pancreatitis and cytokines: "the second attack" by septic complication leads to organ failure," Pancreas, vol. 16, no. 3, pp. 312-315, 1998. 
[15] M. A. Bird, D. Black, P. A. Lange, C. M. Samson, M. Hayden, and K. E. Behrns, "NF $\kappa$ B inhibition decreases hepatocyte proliferation but does not alter apoptosis in obstructive jaundice," Journal of Surgical Research, vol. 114, no. 2, pp. 110117, 2003.

[16] M. A. Bird, P. A. Lange, L. W. Schrum, J. W. Grisham, R. A. Rippe, and K. E. Behrns, "Cholestasis induces murine hepatocyte apoptosis and DNA synthesis with preservation of the immediate-early gene response," Surgery, vol. 131, no. 5, pp. 556-563, 2002.

[17] T.-Z. Liu, K.-T. Lee, C.-L. Chern, J.-T. Cheng, A. Stern, and L.Y. Tsai, "Free radical-triggered hepatic injury of experimental obstructive jaundice of rats involves overproduction of proinflammatory cytokines and enhanced activation of nuclear factor $\kappa \mathrm{B}$," Annals of Clinical and Laboratory Science, vol. 31, no. 4, pp. 383-390, 2001.

[18] J. A. Clemens, D. T. Stephenson, T. Yin, E. B. Smalstig, J. A. Panetta, and S. P. Little, "Drug-induced neuroprotection from global ischemia is associated with prevention of persistent but not transient activation of nuclear factor- $\kappa \mathrm{B}$ in rats," Stroke, vol. 29, no. 3, pp. 677-682, 1998.

[19] P. Ypsilantis, I. Tentes, M. Lambropoulou, et al., "Prophylaxis with mesna prevents oxidative stress induced by ischemia reperfusion in the intestine via inhibition of nuclear factor$\kappa \mathrm{B}$ activation," Journal of Gastroenterology and Hepatology, vol. 23, no. 2, pp. 328-335, 2008.

[20] A. Giakoustidis, D. Giakoustidis, K. Koliakou, et al., "Inhibition of intestinal ischemia/repurfusion induced apoptosis and necrosis via down-regulation of the NF- $\kappa \mathrm{B}, \mathrm{c}$-Jun and caspace3 expression by epigallocatechin-3-gallate administration," Free Radical Research, vol. 42, no. 2, pp. 180-188, 2008.

[21] C. S. Potten, "Epithelial cell growth and diferentiation II, Intestinal aoptosis," American Journal of Physiology, vol. 273, no. 2, pp. 253-257, 1997.

[22] K. Fukuyama, R. Iwakiri, T. Noda, et al., "Apoptosis induced by ischemia-reperfusion and fasting in gastric mucosa compared to small intestinal mucosa in rats," Digestive Diseases and Sciences, vol. 46, no. 3, pp. 545-549, 2001.

[23] S. An, Y. Hishikawa, and T. Koji, "Induction of cell death in rat small intestine by ischemia reperfusion: differential roles of Fas/Fas ligand and Bcl-2/Bax systems depending upon cell types," Histochemistry and Cell Biology, vol. 123, no. 3, pp. 249-261, 2005.

[24] B. A. Jones and G. J. Gores, "Physiology and pathophysiology of apoptosis in epithelial cells of the liver, pancreas, and intestine," American Journal of Physiology, vol. 273, no. 6, part 1, pp. G1174-G1188, 1997.

[25] H. Itoh, M. Yagi, S. Fushida, et al., "Activation of immediate early gene, c-fos, and c-jun in the rat small intestine after ischemia/reperfusion," Transplantation, vol. 69, no. 4, pp. 598604, 2000.

[26] Z. Sun, X. Wang, X. Deng, et al., "Phagocytic and intestinal endothelial and epithelial barrier function during the early stage of small intestinal ischemia and reperfusion injury," Shock, vol. 13, no. 3, pp. 209-216, 2000.

[27] K. Olanders, Z. Sun, A. Börjesson, et al., "The effect of intestinal ischemia and reperfusion injury on ICAM-1 expression, endothelial barrier function, neutrophil tissue influx, and protease inhibitor levels in rats," Shock, vol. 18, no. 1, pp. 8692, 2002.

[28] Z. Sun, X. Wang, R. Wallen, et al., "The influence of apoptosis in intestinal barrier integrity in rats," Scandinavian Journal of Gastroenterology, vol. 33, no. 4, pp. 415-422, 1998.
[29] P. Sileri, S. Morini, G. S. Sica, et al., "Bacterial translocation and intestinal morphological findings in jaundiced rats," Digestive Diseases and Sciences, vol. 47, no. 4, pp. 929-934, 2002.

[30] Y. Yang and M.-Z. Chen, "Inhibitory effect of total extract of Astragalus on hepatocyte apoptosis," Chinese Journal of Pharmacology and Toxicology, vol. 15, no. 4, pp. 287-292, 2001.

[31] L. Zhang, D. Xing, and M. Chen, "Bim $\mathrm{L}$ displacing Bcl- $\mathrm{x}_{\mathrm{L}}$ promotes Bax translocation during TNF $\alpha$-induced apoptosis," Apoptosis, vol. 13, no. 7, pp. 950-958, 2008.

[32] H. J. Kim, S. Y. Kim, J. Kim, et al., "Hepatitis B virus X protein induces apoptosis by enhancing translocation of Bax to mitochondria," IUBMB Life, vol. 60, no. 7, pp. 473-480, 2008.

[33] I. Lang-Rollin, M. Maniati, O. Jabado, et al., "Apoptosis and the conformational change of Bax induced by proteasomal inhibition of PC12 cells are inhibited by Bcl- $\mathrm{x}_{\mathrm{L}}$ and Bcl-2," Apoptosis, vol. 10, no. 4, pp. 809-820, 2005.

[34] G. Gomez, H.-M. Lee, Q. He, E. W. Englander, T. Uchida, and G. H. Greeley Jr., "Acute pancreatitis signals activation of apoptosis-associated and survival genes in mice," Experimental Biology and Medicine, vol. 226, no. 7, pp. 692-700, 2001.

[35] K. G. Wolter, Y.-T. Hsu, C. L. Smith, A. Nechushtan, X.-G. $\mathrm{Xi}$, and R. J. Youle, "Movement of Bax from the cytosol to mitochondria during apoptosis," Journal of Cell Biology, vol. 139, no. 5, pp. 1281-1292, 1997.

[36] A. Gross, J. M. McDonnell, and S. J. Korsmeyer, "Bcl-2 family members and the mitochondria in apoptosis," Genes and Development, vol. 13, no. 15, pp. 1899-1911, 1999.

[37] C. M. Coopersmith, D. O’Donnell, and J. I. Gordon, "Bcl2 inhibits ischemia-reperfusion-induced apoptosis in the intestinal epithelium of transgenic mice," American Journal of Physiology, vol. 276, no. 3, part 1, pp. G677-G686, 1999.

[38] N. S. Cheung, P. M. Beart, C. J. Pascoe, C. A. John, and O. Bernard, "Human Bcl-2 protects against AMPA receptormediated apoptosis," Journal of Neurochemistry, vol. 74, no. 4, pp. 1613-1620, 2000. 


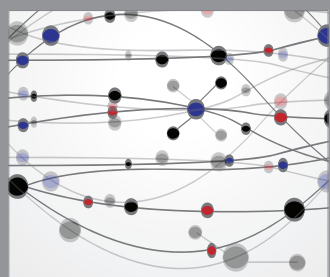

The Scientific World Journal
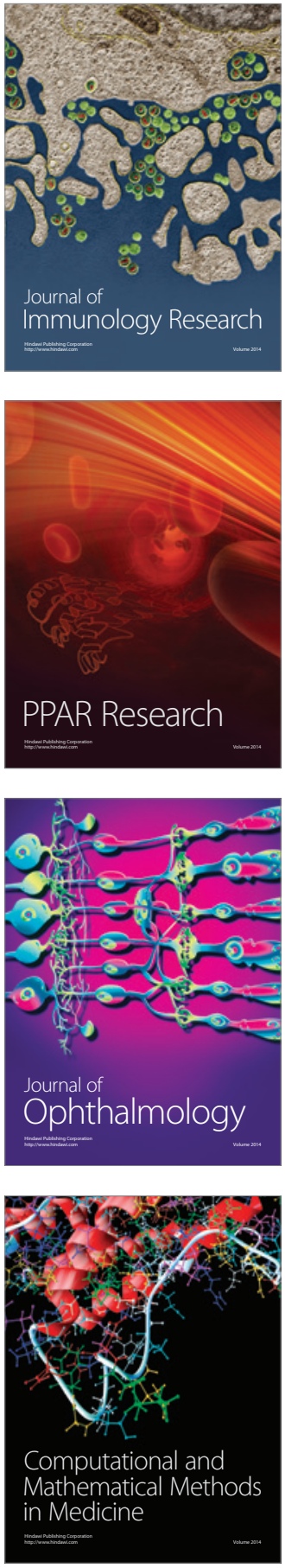

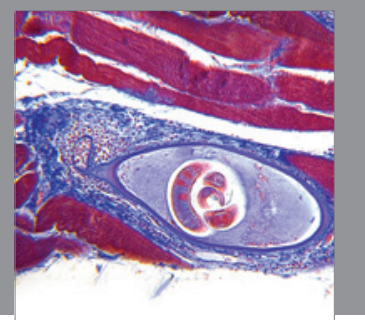

Gastroenterology

Research and Practice
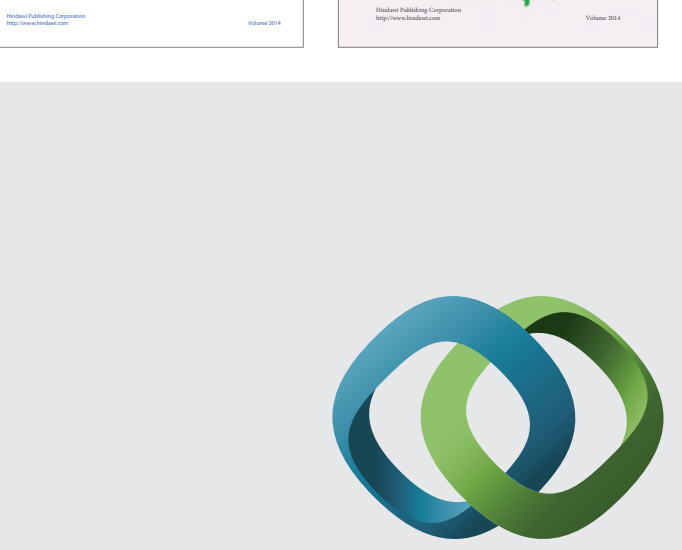

\section{Hindawi}

Submit your manuscripts at

http://www.hindawi.com
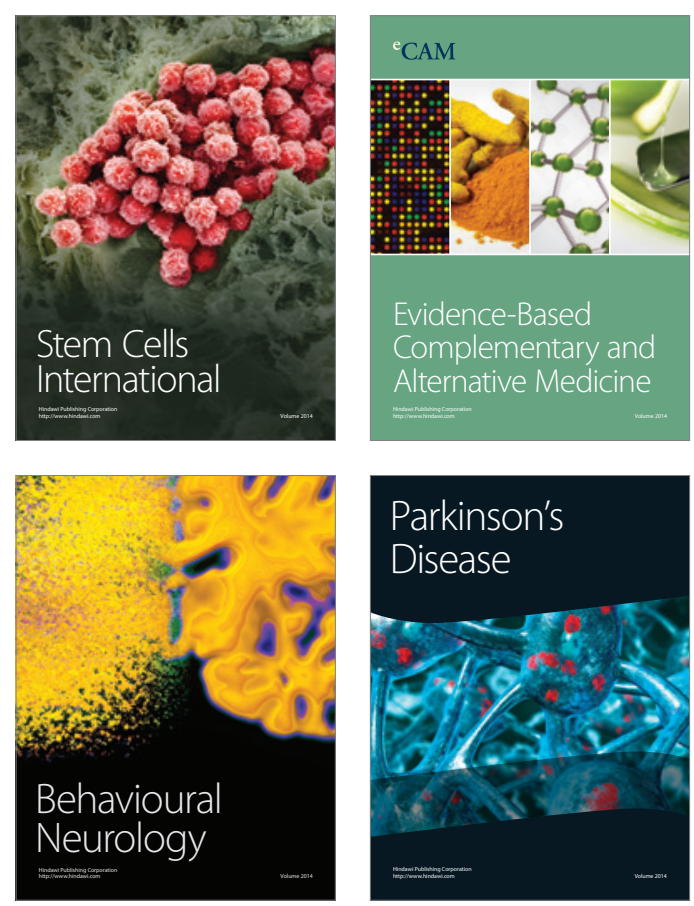

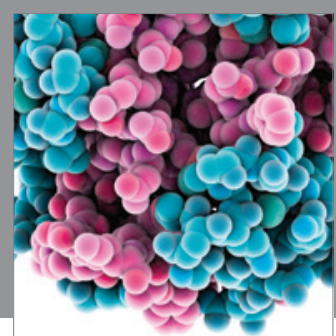

Journal of
Diabetes Research

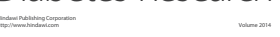

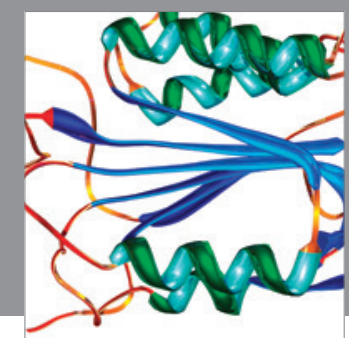

Disease Markers
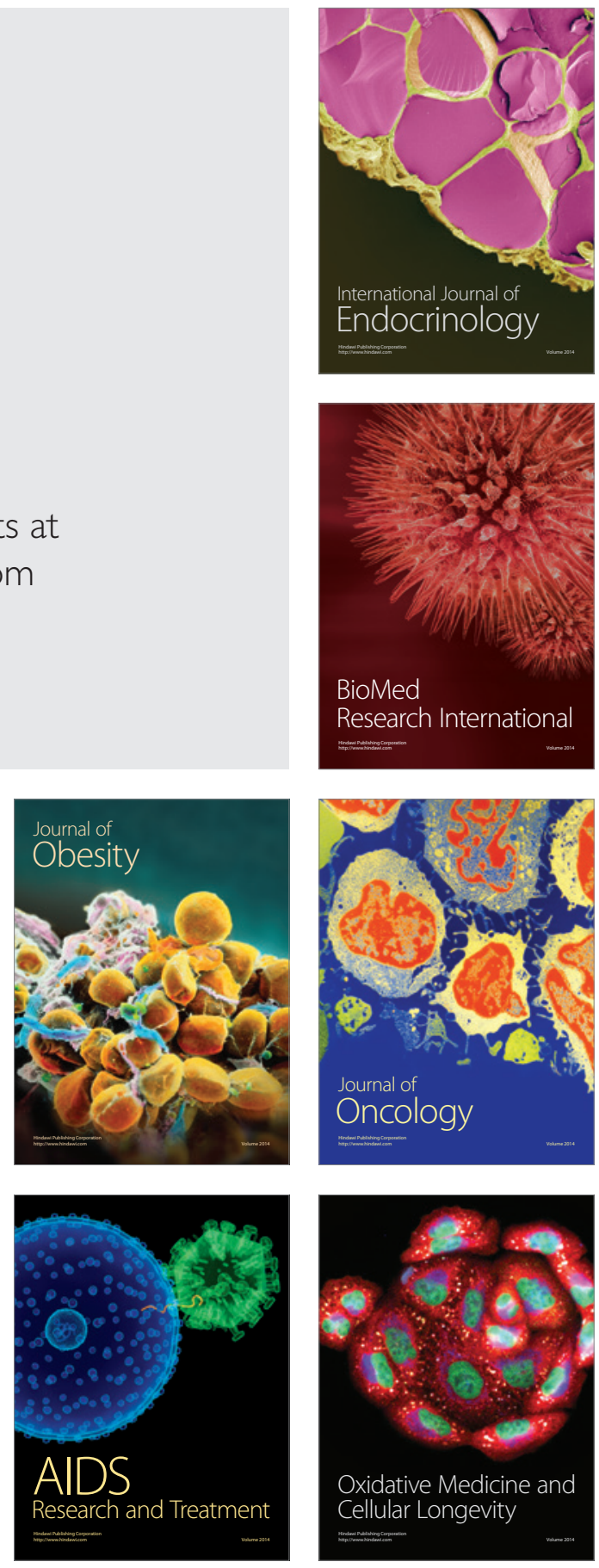